Pathologe 2022 • 43:99-104

https://doi.org/10.1007/s00292-022-01053-9

Angenommen: 2. Januar 2022

Online publiziert: 8. Februar 2022

(c) Der/die Autor(en) 2022

Schwerpunktherausgeber

L. Bubendorf, Basel, Schweiz

\section{In diesem Beitrag}

- Immunzytochemie

- Molekulare Diagnostik mittels Mehrfach-Fluoreszenz-in-situ-Hybridisierung

- Zukunftsausblick-Molekulare Diagnostik mittels Next Generation Sequencing

\title{
Diagnostische und prädiktive Marker in der Harntraktzytologie
}

Tatjana Vlajnic · Lukas Bubendorf

Pathologie, Institut für medizinische Genetik und Pathologie, Universitätsspital Basel, Basel, Schweiz

\section{Zusammenfassung}

In der Routinediagnostik spielt die Mehrfach-Fluoreszenz-in-situ-Hybridisierung (FISH) nach wie vor die führende Rolle in der Abklärung unklarer Atypien in der Harntraktzytologie. Die Paris-Klassifikation (The Paris System, TPS) bildet eine wichtige Grundlage zur gezielten Indikationsstellung der FISH und untermauert die Bedeutung der morphologischen Korrelation für eine integrative Diagnosestellung. Die NextGeneration-Sequencing-Technologie, welche durch gleichzeitigen Nachweis multipler genetischer Alterationen eine hohe Sensitivität erzielt, wird in naher Zukunft auch in der Harntraktzytologie Anwendung finden.

\section{Schlüsselwörter}

Differenzialdiagnose $\cdot$ Hochdurchsatz-Nukleotidsequenzierung $\cdot$ Immunhistochemie ·

Immuncheckpoint-Inhibitoren. Urologische Tumoren

Die Zytologie besitzt einen zentralen Stellenwert bei der Abklärung von Erkrankungen des Harntraktes und in der Nachsorge von Patienten mit bekannten urothelialen Neoplasien. Ihre Stärke liegt zum einen in der hohen Zuverlässigkeit für die Diagnose eines potenziell lebensbedrohlichen high-grade Urothelkarzinoms (HGUC), zum anderen in der relativ einfachen und wenig invasiven Methodik zur Gewinnung von Untersuchungsmaterial, entweder aus der Spülflüssigkeit im Rahmen einer Endoskopie oder auch aus Spontanurin.

Vor einigen Jahren wurde durch die Einführung der Paris-Klassifikation (The Paris System, TPS) eine internationale Standardisierung in der Befundung von Urinzytologien ermöglicht $[1,2]$. Neben der zuverlässigen Diagnose bzw. dem Ausschluss eines HGUC liegt ein weiterer Schwerpunkt dieser Klassifikation darin, die Häufigkeit der bislang uneinheitlich genutzten Diagnose von „Atypien“ zu verringern und so die Aussagekraft der Urinzytologie zu verbessern. Basierend auf den strikt definierten morphologischen Kriterien der ParisKlassifikation lassen sich eindeutig negati- ve oder eindeutig maligne Befunde in der Mehrzahl der Fälle als solche erkennen. Ein Teil bleibt dennoch morphologisch unklar und erfordert Zusatzmethoden zur definitiven Klärung (•Tab. 1). Trotz erheblicher und kontinuierlicher Anstrengungen über die letzten beiden Jahrzehnte haben sich bisher nur wenige Biomarker und Methoden der Urinzytologie in der klinische Routine zur verbesserten Diagnostik und/ oder Verlaufskontrolle als nützlich erwiesen [3-5]. Zunehmend wächst auch die Nachfrage nach prädiktiven Markern beim Urothelkarzinom in Zusammenhang mit personalisierter Medizin.

\section{Immunzytochemie}

Immunzytochemische Untersuchungen in der Urinzytologie wurden im diagnostischen Alltag bislang vor allem für differenzialdiagnostische Fragestellungen bzw. für die Abgrenzung des Urothelkarzinoms von Manifestationen anderer Tumoren eingesetzt. Grundsätzlich sollte die Immunzytochemie direkt an Ausstrichpräparaten/ Zytospins etabliert werden, da eine Anfertigung von Zellblöcken in der Urinzytologie unüblich ist. $\mathrm{Zu}$ den gebräuch- 
Tab. 1 Atypische urotheliale Zellen (AUC) Hauptkriterium (erforderlich):

Nichtsuperfizielle und nichtdegenerativ veränderte Urothelien mit Kern-PlasmaRelation $>0,5$

Nebenkriterien (mindestens eines):

Geringe Hyperchromasie

Kernmembran: Unregelmäßigkeiten (Kontur und Dicke)

Chromatin: irregulär, vergröbert, verklumpt

lichsten Markern zum Nachweis urothelialen Ursprungs von malignen Epithelzellen in der Harntraktzytologie zählen GATA3, CK7, CK20 und p40 oder p63 [6]. Die weitaus häufigste Differenzialdiagnose ist das Adenokarzinom der Prostata. Falls die typischen morphologischen Charakteristika wie azinäre Strukturen und prominente Nukleolen fehlen, kann NKX3.1 als relativ spezifischer und sensitiver Marker für Karzinome prostatischen Ursprungs eingesetzt werden ([7]; • Abb. 1). Im Gegensatz zum Urothelkarzinom sind Adenokarzinome der Prostata zudem praktisch immer negativ für die urothelialen Marker CK7, GATA3 und p40/p63 [8]. Basalzellkarzinome des Prostata mit aberranter p63Expression sind eine Rarität [9]. Als weiterer spezifischer Marker für Prostatakarzinome gilt PSA, welches jedoch bei wenig differenzierten Prostatakarzinomen lediglich schwach oder gar nicht exprimiert wird. Falls nur wenige Präparate für eine immunzytochemische Untersuchung zur Verfügung stehen, ist eine Priorisierung der beiden Marker NKX3.1 und CK7 sinnvoll. CK7 wird in Urothelkarzinomen praktisch immer (87-100\%) exprimiert [10]. Eine fokale CK7-Positivität wurde in bis zu $10 \%$ der Adenokarzinome der Prostata beschrieben [11]. Nach unserer Erfahrung wird dieser Marker bei Prostatakarzinomen jedoch nie diffus exprimiert. Eine einfache immunhistochemische Bestimmung des molekularen Subtyps (v.a. basal und luminal) mittels geeigneter Marker (z.B. CK5/6 oder p63 und CK20 oder GATA3) wäre zwar grundsätzlich interessant und potenziell relevant, gilt aber derzeit noch nicht als Standard [12-14] und hätte in der Harntraktzytologie nur eine untergeordnete oder keine praktische Bedeutung.

Eine weitere Anwendung der Immunzytochemie wurde erst kürzlich auf potenzielle prädiktive Marker erweitert. Inaktivierende Mutationen im ARID1AGen wurden mit reduziertem Ansprechen auf Bacillus-Calmette-Guérin(BCG)-Therapie in Verbindung gebracht [15]. Eine Studie zeigte, dass ein Expressionsverlust von ARID1A in der Immunzytochemie als verlässlicher Surrogatmarker für ARID1AMutationen dient ([16]; • Abb. 2). Die immunzytochemische Untersuchung der PDL1-Expression zur Selektion von Patienten für eine Behandlung mit Immuncheckpoint-Inhibitoren (ICI) ist an Papanicolaou-gefärbten zytologischen Präparaten oder Zellblockpräparaten grundsätzlich möglich [17]. Allerdings können an zytologischen Präparaten nur Tumorzellen gewertet werden, sodass nur eine Bestimmung des PD-L1-Tumor-Proportion-Score (TPS) möglich ist. Eine Aussage über die Expression an Immunzellen und den PDL1-Combined-Positive-Score (CPS) ist nur eingeschränkt möglich, da sich die tumorassoziierten Immunzellen ohne Gewebekontext in der Zytologie nicht auswerten lassen. Bei einem Anteil PD-L1-positiver Tumorzellen (TPS) von > 10\%, liegt der CPS jedoch ungeachtet der tumorassoziierten Immunzellen definitionsgemäß ebenfalls bei über 10, was mit einem Ansprechen auf den Immuncheckpoint-Inhibitor (ICI) Pembrolizumab assoziiert ist. Die PD-L1-Testung in der Harntraktzytologie stellt im Alltag aber keine Notwendigkeit dar, da bei fortgeschrittenen Urothelkarzinomen praktisch immer ausreichend histologisches Material für eine PD-L1Testung mit Bestimmung des CPS zur Verfügung steht [18]. Ein Verlust der MTAPExpression als Surrogatmarker für eine homozygote 9p21-Deletion wurde kürzlich als potenziell interessanter negativer prädiktiver Marker für das Ansprechen auf ICI bei verschiedenen malignen Tumoren inkl. dem Urothelkarzinom vorgeschlagen [19]. Auch hier dürfte in der Zukunft die Untersuchung an Gewebeproben im Vordergrund stehen, zumal ethanolfixierte zytologische Präparate für die MTAP-Immunzytochemie nach unserer Erfahrung ungeeignet sind (• Abb. 3).

\section{Molekulare Diagnostik mittels Mehrfach-Fluoreszenz-in-situ- Hybridisierung}

Für den Nachweis chromosomaler Aberration an zytologischen Präparaten eignet sich am besten eine Fluoreszenz-in-situHybridisierung (FISH). Der kommerziell erhältliche und von der U.S: Food and Drug Administration (FDA) zugelassene Mehrfach-FISH-Test UroVysion ${ }^{\text {TM }}$ (Abbott Laboratories, Abbott Park, IL, USA) bleibt nach wie vor eine der am besten etablierten Methoden zur Abklärung von Atypien in der Urinzytologie [20-23]. Dieser FISHTest besteht aus 4 Sonden für die Chromosomen 3, 7, 17 und den 9p21-Lokus. Ein ähnlicher Quadrupel-FISH-Test eines anderen Herstellers ist verfügbar, wurde bisher aber nicht in größeren publizierten Studien verwendet $\left(Z y\right.$ toLight ${ }^{\circledR}$ Bladder Cancer Quadruple Color Probe, ZytoVision $\mathrm{GmbH}$, Bremerhaven, Deutschland). Der Nachweis von unbalancierten numerischen chromosomalen Aberrationen in mindestens 2 Chromosomen und/oder ein kompletter oder relativer Verlust von 9p21 (entsprechend einer homozygoten oder heterozygoten 9p21-Deletion) sind diagnostisch für eine urotheliale Neoplasie und schließen reaktive Veränderungen mit praktischer Sicherheit aus [24]. Eine Ausnahme stellt das tetraploide oder oktaploide Muster dar (jeweils 4 bzw. 8 Signale von jeder Sonde), das auch bei ausgeprägt reaktiv veränderten Urothelien vorkommen kann. Dessen Nachweis sollte deshalb, insbesondere wenn nur in wenigen Zellen, nicht als eindeutig positives FISH-Resultat interpretiert werden [24]. Sensitivität und Spezifität dieser Untersuchung hängen stark von Erfahrung und Expertise des Untersuchers und von der zytologischen Diagnose ab. Dabei bieten die Paris-Kategorien eine wichtige Grundlage zur gezielten Indikationsstellung. Vor deren Hintergrund lässt sich die FISH-Untersuchung vor allem bei den Kategorien AUC (atypische urotheliale Zellen) und SHGUC (Verdacht auf high-grade Urothelkarzinom) sinnvoll in den diagnostischen Algorithmus implementieren ([24, 25]; - Abb. 4). Wir haben kürzlich gezeigt, dass unsere Zytologien mit AUC oder SHGUC gemäß TPS in jeweils $46 \%$ bzw. $84 \%$ FISH-positiv waren, was in diesen 


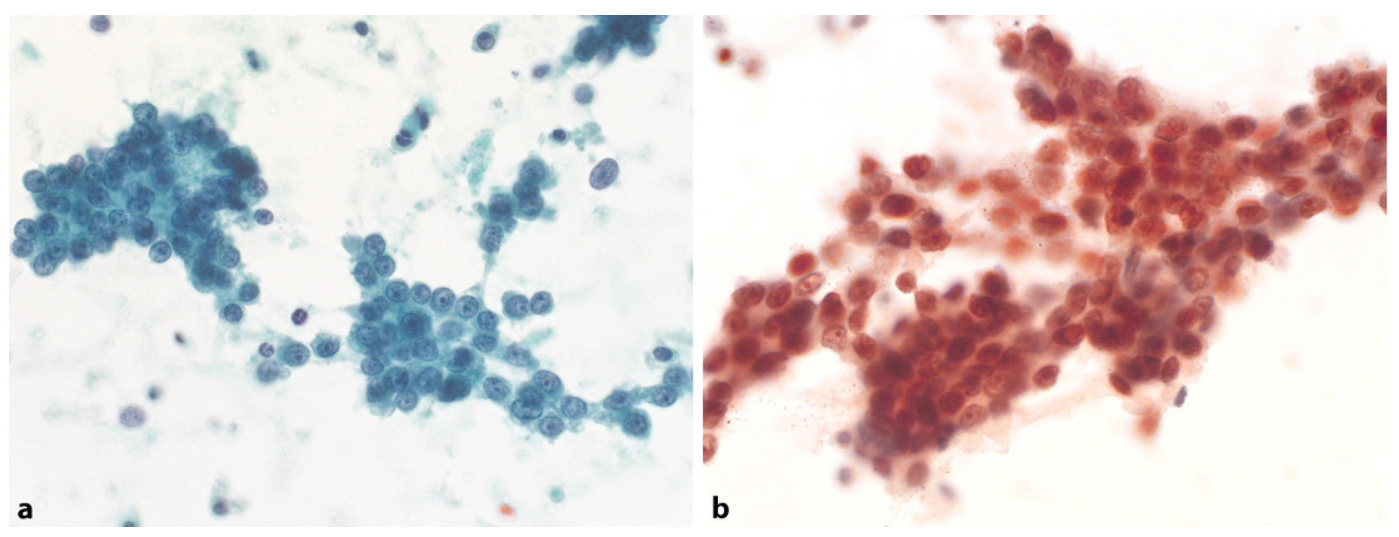

Abb. $1<$ Zellen eines Adenokarzinoms der Prostata in einer Spülzytologie der Harnblase. a PapanicolaouFärbung, b immunzytochemisch nukleär positiv für NKX3.1. Vergr. jeweils 630:1

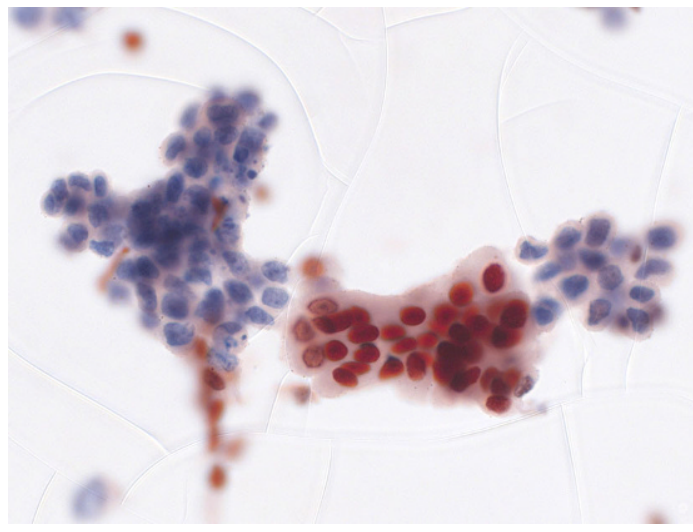

(20-23\%), KDM6A (26-48\%) und ARID1A (10-20\%) sind beim Urothelkarzinom je nach Stadium und Lokalisation besonders häufig mutiert und daher in molekularen Assays oft vertreten [30, 31]. Einige der häufigen Genmutationen haben prognostische Implikationen und wurden z. B. mit Tumorgrad und Stadium bei Präsentation assoziiert, andere korrelieren mit Therapieansprechen [5]. Insbesondere FGFR-Alterationen, die in ca. $15 \%$ der fortgeschrittenen Urothelkarzinome vorkommen, gelten als prädiktiv für das Ansprechen auf FGFR-Inhibitoren [14]. Dank des technischen Fortschritts in den letzten Jahren ist die klassische Sanger-Sequenzierung zunehmend durch neue Sequenziertechniken (Next Generation Sequencing, NGS) ersetzt worden. NGS erlaubt nicht nur den Nachweis von Mutationen und Fusionen, sondern auch von epigenetischen Veränderungen (DNA-Methylierung) und Kopienzahlveränderungen und bietet den Vorteil, dass diese gleichzeitig nachgewiesen werden können. Allerdings ist anzumerken, dass RNA-basierte Untersuchungen zum Nachweis von Genfusionen aus zytologischen Präparaten je nach Fixierungsmethode und Menge an Tumorzellen schwieriger sein können als aus Paraffinblöcken. NGS-basierte Analysen von zytologischen Präparaten des Harntrakts versprechen somit eine hohe Sensitivität und werden zukünftig wahrscheinlich eine Anwendung im diagnostischen Alltag finden. Als gutes Beispiel dient der UroSEEK-Assay am Urin, bei dem 11 ausgewählte Gene auf Mutationen oder Veränderungen der Kopienzahl untersucht werden. Dieser Test wurde für die Nachkontrolle von Patienten mit resezierten Urothelkarzinomen und für die Abklärung von zytologischen Atypien ent- 

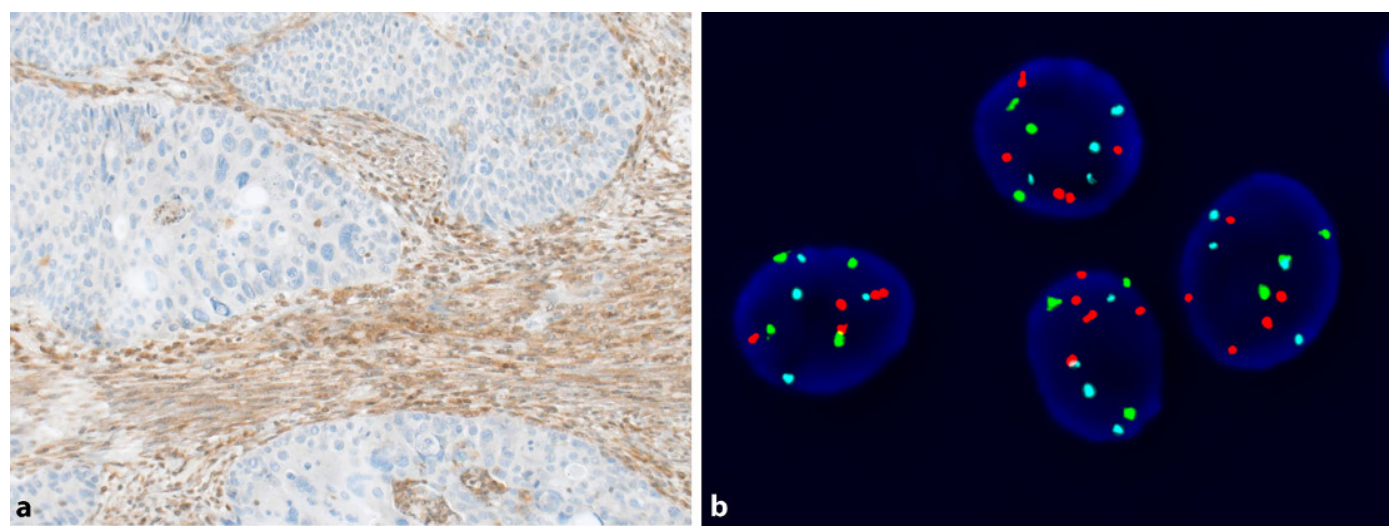

Abb. $3 \varangle$ a Invasives highgrade Urothelkarzinom mit Verlust der MTAP Expression. Vergr. 200:1. b FISH an zuvor Papanicolaougefärbtem zytologischem Präparat: Zellen eines highgrade Urothelkarzinoms mit Vermehrung der Chromosomen 3 (rot), 7 (grün) und 17 (blau) bei vollständigem Fehlen der 9p21Signale (gold; homozygote 9p21-Deletion). Vergr. 1000:1
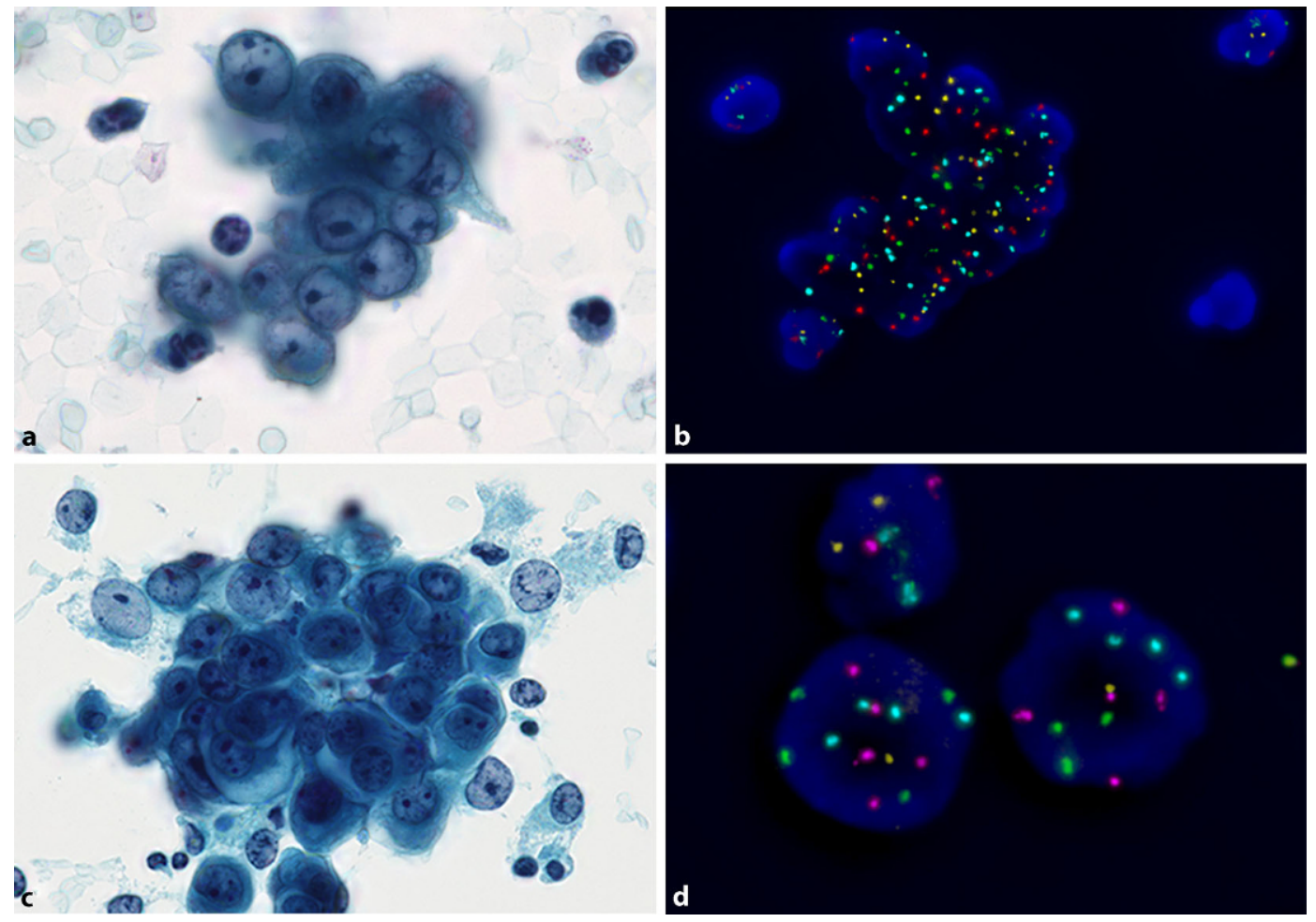

Abb. 4 ム Fluorenszen-in-situ-Hybridisierung (FISH) an Papanicolaou-gefärbten zytologischen Präparaten zur Abklärung der Diagnose „atypische urotheliale Zellen (AUC)“. Atypische Urothelzellen (a) mit negativem FISH-Resultat (b, jeweils 2 Signale für die Chromosomen 3, 7 und 17 und für 9p21 pro Zellkern), vereinbar mit reaktiven Zellveränderungen. Vergr. jeweils 630:1. Klinischer Nachweis von Harnwegssteinen. Atypische Urothelien (c, Vergr. 400:1) mit deutlich positivem FISH-Befund (d, unregelmäßige Vermehrung für die Chromosomen 3,7 und 17 mit einem relativen Verlust für 9p21), beweisend für eine urotheliale Neoplasie und verdächtig auf high-grade Urothelkarzinom. Anschließend histologische Diagnose eines Carcinoma in situ. Vergr. 1000:1

wickelt [28, 32, 33]. In einer retrospektiven Studie zeigte er vor allem bei low-grade nichtinvasiven papillären Urothelkarzinomen eine hohe Sensitivität im Vergleich zur Zytologie [32]. Allerdings ist für die Diagnose von low-grade nichtinvasiven papillären Urothelkarzinomen, die zystoskopisch gut sichtbar sind, die Indikation fragwürdig, da der Test in Relation zu den hohen Kosten wenig klinisch relevante Zusatzinformationen liefert. In einer Follow-up-
Kohorte von Patienten mit "Atypien" in der Harntraktzytologie (AUC- und SHGUCKategorien zusammengenommen) erwies sich der UroSEEK-Assay mit einer Sensitivität von $74 \%$ und einem negativen prädiktiven Wert von $53 \%$ als weniger robust [33]. Die wichtigste Voraussetzung für die NGS-Analyse ist eine ausreichende Anzahl bzw. ein relativer Anteil an Tumorzellen von mind. $2 \%$ in der zytologischen Probe. Daher bieten sich insbesondere zellreiche
Urinzytologieproben für eine NGS-Testung an. In einer kürzlich veröffentlichten Übersichtsarbeit zur Rolle des NGS in der Urinzytologie wurde vorgeschlagen, die Urinzytologie als Triage zu benutzen. Somit ließe sich beurteilen, welche Präparate für eine direkte NGS-Testung geeignet sind und bei welchen die Tumorzellen, z. B mittels Laser-Mikrodissektion, angereichert werden müssten [5]. Obwohl NGS zweifellos eine vielversprechende Methode in der 
Harntraktzytologie darstellt und mit weiteren technischen Fortschritten auf diesem Gebiet zu rechnen ist, sind umfassende Studien zur Festlegung der Wertigkeit und der optimalen Indikationen notwendig. Neben technischen Faktoren muss vor allem auch das Kosten-Nutzen-Verhältnis kritisch untersucht werden. Ökonomische Interessen an einer breiten Anwendung sollten dem medizinischen Zusatzgewinn im Vergleich zu einer standardisierten zytologischen Diagnose gegenübergestellt werden.

\section{Fazit für die Praxis}

- Die Morphologie besitzt nach wie vor eine zentrale Bedeutung in der Harntraktzytologie und hat durch die standardisierte Befundung im Rahmen des Paris-Systems (TPS) an Bedeutung gewonnen.

- Die Immunzytochemie ist in ausgewählten Fällen nützlich für eine diagnostische Zuordnung von Tumorzellen und kann auch einen potenziell prädiktiven Wert besitzen.

- Die Fluoreszenz-in-situ-Hybridisierung ist bei unklaren Befunden der TPS-Kategorien AUC (atypische urotheliale Zellen) oder SHGUC (Verdacht auf high-grade Urothelkarzinom) diagnostisch hilfreich.

- Next Generation Sequencing ist eine erfolgversprechende Zusatzmethode für die Harntraktzytologie, deren praktischer Wert für die verschiedenen zytologischen Kategorien und klinischen Situationen aber kritisch hinterfragt und noch weiter untersucht werden muss.

\section{Korrespondenzadresse}

\section{PD Dr. med. Tatjana Vlajnic}

Pathologie, Institut für medizinische Genetik und Pathologie, Universitätsspital Basel Schönbeinstr. 40, 4031 Basel, Schweiz Tatjana.Vlajnic@usb.ch

Funding. Open access funding provided by University of Basel

\section{Einhaltung ethischer Richtlinien}

Interessenkonflikt. T. Vlajnic und L. Bubendorf geben an, dass kein Interessenkonflikt besteht.

Für diesen Beitrag wurden von den Autoren keine Studien an Menschen oder Tieren durchgeführt. Für die aufgeführten Studien gelten die jeweils dort angegebenen ethischen Richtlinien.

Open Access. Dieser Artikel wird unter der Creative
Commons Namensnennung 4.0 International Lizenz veröffentlicht, welche die Nutzung, Vervielfältigung, Bearbeitung, Verbreitung und Wiedergabe in jeglichem Medium und Format erlaubt, sofern Sie den/die ursprünglichen Autor(en) und die Quelle ordnungsgemäßnennen, einen Link zur Creative Commons Lizenz beifügen und angeben, ob Änderungen vorgenommen wurden.

Die in diesem Artikel enthaltenen Bilder und sonstiges Drittmaterial unterliegen ebenfalls der genannten Creative Commons Lizenz, sofern sich aus der Abbildungslegende nichts anderes ergibt. Sofern das betreffende Material nicht unter der genannten Creative Commons Lizenz steht und die betreffende Handlung nicht nach gesetzlichen Vorschriften erlaubt ist, ist für die oben aufgeführten Weiterverwendungen des Materials die Einwilligung des jeweiligen Rechteinhabers einzuholen.

Weitere Details zur Lizenz entnehmen Sie bitte der Lizenzinformation auf http://creativecommons.org/ licenses/by/4.0/deed.de.

\section{Literatur}

1. Rosenthal DL, Wojcik EM, Kurtycz DF (2016) The Paris system for reporting urinary cytology, 1. Aufl. Springer, Berlin

2. Savic S, Vlajnic T, Bubendorf L (2017) The Paris system for classification of urinary cytology. Pathologe 38(5):451-462

3. Sugeeta SS, Sharma A, Ng K, Nayak A, Vasdev N (2021) Biomarkers in bladder cancer surveillance. Front Surg 8:735868

4. Henning GM, Barashi NS, Smith ZL (2021) Advances in biomarkers for detection, surveillance, and prognosis of bladder cancer. Clin Genitourin Cancer 19(3):194-198

5. Harris T, Sheel A, Zong $Y$, Hutchinson $L M$, Cornejo KM, Bubendorf L et al (2021) Cytologically targeted next-generation sequencing: a synergy for diagnosing urothelial carcinoma. J Am Soc Cytopathol 10(1):94-102

6. Akgul M, MacLennan GT, Cheng L (2020) The applicability and utility of immunohistochemical biomarkers in bladder pathology. Hum Pathol 98:32-55

7. Gurel B, Ali TZ, Montgomery EA, Begum S, Hicks J, Goggins M et al (2010) NKX3.1 as a marker of prostatic origin in metastatic tumors. Am J Surg Pathol 34(8):1097-1105

8. Epstein Jl, Egevad L, Humphrey PA, Montironi R, Members of the ISUP Immunohistochemistry in Diagnostic Urologic Pathology Group (2014) Best practices recommendations in the application of immunohistochemistry in the prostate: report from the international society of urologic pathology consensus conference. Am J Surg Pathol 38(8):e6-e19

9. Ali TZ, Epstein Jl (2007) Basal cell carcinoma of the prostate: a clinicopathologic study of 29 cases. Am J Surg Pathol 31(5):697-705

10. Amin MB, Trpkov K, Lopez-Beltran A, Grignon D, Members of the ISUP Immunohistochemistry in Diagnostic Urologic Pathology Group (2014) Best practices recommendations in the application of immunohistochemistry in the bladder lesions: report from the international society of urologic pathology consensus conference. Am JSurg Pathol 38(8):e20-34
11. Bassily NH, Vallorosi CJ, Akdas G, Montie JE, Rubin MA (2000) Coordinate expression of cytokeratins 7 and 20 in prostate adenocarcinoma and bladder urothelial carcinoma. Am J Clin Pathol 113(3):383-388

12. Warrick Jl, Knowles MA, Yves A, van der Kwast T, Grignon DJ, Kristiansen $G$ et al (2020) Report from the international society of urological pathology (ISUP) consultation conference on molecular pathology of urogenital cancers. II. Molecular pathology of bladder cancer: progress and challenges. Am J Surg Pathol 44(7):e30-e46

13. Guo CC, Bondaruk J, Yao H, Wang Z, Zhang L, Lee $S$ et al (2020) Assessment of luminal and basal phenotypes in bladder cancer. Sci Rep 10(1):9743

14. Schulz GB, StiefCG, Saar M, Vogeli TA, Todenhofer T, Knuchel R et al (2021) Molecular diagnostics of bladder cancer-practical ramifications. Urologe A 60(10):1349-1358

15. Pietzak EJ, Bagrodia A, Cha EK, Drill EN, lyer G, Isharwal Setal (2017) Next-generation sequencing of nonmuscle invasive bladder cancer reveals potential biomarkers and rational therapeutic targets. Eur Urol 72(6):952-959

16. Dugas SG, Muller DC, Le Magnen C, FedererGsponer J, Seifert HH, Ruiz C et al (2019) Immunocytochemistry for ARID1A as a potential biomarker in urine cytology of bladder cancer. Cancer 127(9):578-585

17. laccarino A, Salatiello M, Migliatico I, De Luca C, Gragnano G, Russo M et al (2021) PD-L1 and beyond: immuno-oncology in cytopathology. Cytopathology 32(5):596-603

18. Reis H, Szarvas T, Grunwald V (2019) Predictive biomarkers in oncologic uropathology. Pathologe 40(3):264-275

19. Han G, Yang G, Hao D, Lu Y, Thein K, Simpson BS et al (2021) 9p21 loss confers a cold tumor immune microenvironment and primary resistance to immune checkpoint therapy. Nat Commun 12(1):5606

20. Halling KC, King W, Sokolova IA, Meyer RG, Burkhardt HM, Halling AC etal (2000) A comparison of cytology and fluorescence in situ hybridization for the detection of urothelial carcinoma. J Urol 164(5):1768-1775

21. Bubendorf $L$ (2011) Multiprobe fluorescence in situ hybridization (UroVysion) for the detection of urothelial carcinoma-FISHing for the right catch Acta Cytol 55(2):113-119

22. Zellweger T, Benz G, Cathomas G, Mihatsch MJ, Sulser T, Gasser TC et al (2006) Multi-target fluorescence in situ hybridization in bladder washings for prediction of recurrent bladder cancer. Int JCancer 119(7):1660-1665

23. Nagai T, Naiki T, Etani T, lida K, Noda Y, Shimizu N et al (2021) UroVysion fluorescence in situ hybridization in urothelial carcinoma: a narrative review and future perspectives. Transl Androl Urol 10(4):1908-1917

24. Bubendorf LCN, Fischer AH, Katz RL, Schmitt $F$, Strojan Fležar M et al (2022) Ancillary studies in urinary cytology. In: Rosenthal DL, Wojcik E, Kurtycz DF (Hrsg) The paris system for reporting urinary cytology, 2. Aufl. Springer, Berlin

25. Vlajnic T, Gut A, Savic S, Bubendorf L (2020) The Paris system for reporting urinary cytology in daily practice with emphasis on ancillary testing by multiprobe FISH.J Clin Pathol 73(2):90-95

26. GlatzK, Willi N, GlatzD, Barascud A, GrilliB, Herzog M et al (2006) An international telecytologic quiz on urinary cytology reveals educational deficits and absence of a commonly used classification system. Am JClin Pathol 126(2):294-301 
27. Avogbe PH, Manel A, Vian E, Durand G, Forey N, Voegele $C$ et al (2019) Urinary TERT promoter mutations as non-invasive biomarkers for the comprehensive detection of urothelial cancer. EBioMedicine 44:431-438

28. Springer SU, Chen $\mathrm{CH}$, Rodriguez Pena MDC, Li L, Douville C, Wang Y et al (2018) Non-invasive detection of urothelial cancer through the analysis of driver gene mutations and aneuploidy. Elife 7:e32143. https://doi.org/10.7554/eLife.32143

29. Ward DG, Gordon NS, BoucherRH, PirrieSJ, BaxterL, Ott $S$ et al (2019) Targeted deep sequencing of urothelial bladder cancers and associated urinary DNA: a 23-gene panel with utility for noninvasive diagnosis and risk stratification. BJU Int 124(3):532-544

30. Glaser AP, Fantini D, Shilatifard A, Schaeffer EM, Meeks JJ(2017) The evolving genomiclandscape of urothelial carcinoma. Nat Rev Urol 14(4):215-229

31. Gao J, Aksoy BA, Dogrusoz U, Dresdner G, Gross B, Sumer SO et al (2013) Integrative analysis of complex cancer genomics and clinical profiles using the cBioPortal. Sci Signal 6(269):pl1

32. Eich ML, Rodriguez Pena MDC, Springer SU, Taheri D, Tregnago AC, Salles DC et al (2019) Incidence and distribution of UroSEEK gene panel in a multi-institutional cohort of bladder urothelial carcinoma. Mod Pathol 32(10):1544-1550

33. Rodriguez Pena MDC, Springer SU, Taheri D, Li L, Tregnago AC, Eich ML et al (2020) Performance of novel non-invasive urine assay UroSEEK in cohorts of equivocal urine cytology. Virchows Arch 476(3):423-429

\section{Diagnostic and predictive markers in urinary tract cytology}

Multiprobe fluorescence in situ hybridization (FISH) still remains the gold standard for clarifying inconclusive atypia in urinary cytology in daily routine practice. The Paris Classification System (The Paris System, TPS) provides an important basis for the specific indication of FISH and emphasizes the importance of morphological correlation for an integrative approach to diagnosis. Next-generation sequencing technology in urinary specimens, which is highly sensitive for simultaneous detection of multiple genetic alterations, is also likely to play a diagnostic role in the near future.

\section{Keywords}

Differential diagnosis · High-throughput nucleotide sequencing · Immunohistochemistry · Immune checkpoint inhibitors · Urologic neoplasms

\section{Bilder sagen mehr als Worte}

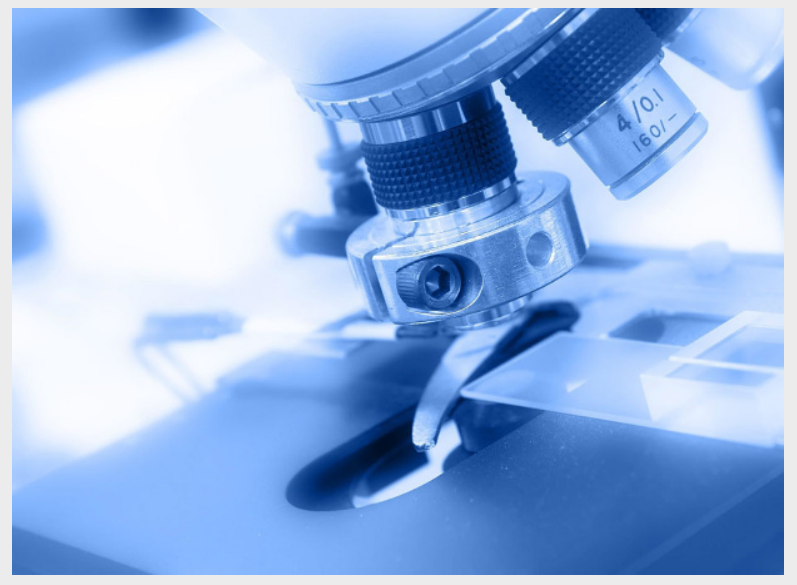

(c) idrutu / Fotolia

Verlag und Herausgeberboard von „Der Pathologe“ laden Sie alle herzlich ein, bei uns Ihre „ganz besonderen“ Bilder zur Publi- kation einzureichen. Manche Bilder sind "ganz besonders", weil sie ein Krankheitsbild absolut prototypisch, klassisch oder exemplarisch zum Ausdruck bringen. Oder weil sie Strukturen, die normalerweise nur schwer oder unvollständig darstellbar sind, ungewöhnlich vollständig und beeindruckend sichtbar machen. Die Abbildungen sollen interessant, lehrreich und für die alltägliche Diagnostik relevant sein. Neben jedem Bild soll ein kurzer Textabschnitt das Wesentliche der Abbildung zusammenfassen.

\section{Manuskript-Hinweise:}

- 1-3 aussagekräftige Bilder

- Aussagekräftiger Manuskript-Titel

- 1 Bildlegende mit max. 2500 Zeichen inkl. Leerzeichen

- Ihre vollständige Korrespondenzadresse

Senden Sie Ihre Bilder und den Kurztext an:

\section{Dagmar Lorenz}

Dagmar.Lorenz@springer.com 